\title{
The Faraday screen near the nucleus of the CSS quasar 3C 138
}

\author{
W. D. Cotton ${ }^{1}$, D. Dallacasa ${ }^{2}$, C. Fanti ${ }^{2,3}$, R. Fanti ${ }^{2,3}$, A. R. Foley ${ }^{4}$, R. T. Schilizzi ${ }^{5,6}$, and R. E. Spencer ${ }^{7}$ \\ 1 National Radio Astronomy Observatory ${ }^{\star}, 520$ Edgemont Road, Charlottesville, VA 22903-2475, USA \\ 2 Istituto di Radioastronomia del CNR, Via P. Gobetti 101, 40129 Bologna, Italy \\ 3 Dipartimento di Fisica, Universitá di Bologna, via Irnerio 46, 40126 Bologna, Italy \\ ${ }^{4}$ Netherlands Foundation for Research in Astronomy, Postbus 2, 7990 AA Dwingeloo, The Netherlands \\ 5 Joint Institute for VLBI in Europe, Postbus 2, 7990 AA Dwingeloo, The Netherlands \\ 6 Sterrenwacht Leiden, Postbus 9513, 2300 RA Leiden, The Netherlands \\ 7 NRAL-Jodrell Bank, University of Manchester, Macclesfield Cheshire, SK11 9DL, UK
}

Received 15 January 2003 / Accepted 6 April 2003

\begin{abstract}
Compact Steep Spectrum (CSS) sources are known to show strong optical line emission (Gelderman \& Whittle 1994) which is generally considered to be due to the interaction between the radio jet and the ISM. This argument is strengthened by the HST observations of Axon et al. (2000) which show this emission is frequently associated with the radio jet. Previous observations of the CSS quasar 3C 138 (Cotton et al. 1997) have shown that the bulk of the jet has very low amounts of Faraday rotation whereas the inner jet shows very high Faraday rotation $\left(\approx-5000 \mathrm{rad} \mathrm{m}^{-2}\right)$. The inner jet is dominated by moving components which allows observing the fine scale of the Faraday screen as the components move behind it. We report the results of such a monitoring campaign which indicated that there is structure in the Faraday screen on the pc and possibly sub pc scale. Motions of the polarized component slowed dramatically during the course of these observations, limiting the region probed. The observations also strongly suggest that the polarized emission from the inner jet is seen through limited holes in a dense Faraday screen. In this case, the observed variations in Faraday rotation are possibly the result of jet-ISM interactions.
\end{abstract}

Key words. polarization - galaxies: quasars: individual: 3C 138 - galaxies: jets - galaxies: nuclei - radio continuum: galaxies

\section{Introduction}

Radio observations of the nuclear regions in Active Galactic Nuclei (AGNs) are a potentially powerful probe of the ionized gas near the nuclei of these objects. A combination of the high angular resolution available to Very Long Baseline Interferometry (VLBI) techniques and relatively easy measurement of the Faraday rotation by the intervening medium of the intrinsically linearly polarized emission of the jet allows a high spatial resolution probe of the ionized gas. Motion of features in the radio jets allow detailed mapping of the Faraday rotation over extended regions.

Taylor et al. (1998), Zavala \& Taylor (2001) and Zavala $\&$ Taylor (2002) have shown large and sometimes variable rotation measures in a number of sources illustrating the utility of this technique. These regions of high Faraday rotation appear confined to the immediate areas of the nucleus. Compact Steep Spectrum Sources (CSS) (Peacock \& Wall 1982) have

Send offprint requests to: W. D. Cotton, e-mail: bcotton@nrao.edu

* The National Radio Astronomy Observatory (NRAO) is operated by Associated Universities Inc., under cooperative agreement with the National Science Foundation. long been known to exist in hosts with unusually large amounts of optical line emission (Gelderman \& Whittle 1994) which is often cited as evidence for enhanced jet-ISM interactions. Axon et al. (2000) give HST observations showing that this emission is largely associated with the radio jet. Extended regions of high rotation measure have been reported in a number of CSS sources (Flatters 1998; Taylor et al. 1998; Nan et al. 1999; Mantovani et al. 2002). See O’Dea (1998) for a recent review of CSS sources.

The CSS quasar 3C 138 was one of the few objects in the study by Axon et al. (2000) which did not show line emission outside an unresolved nuclear region. This is in agreement with our previously reported results (Cotton et al. 1997) which found a very low rotation measure along the bulk of the jet but very high Faraday rotation close to the nucleus. In addition, this polarized component in the inner jet is moving (Cotton et al. 1997; Shen et al. 2001), allowing measurements of the Faraday rotation over an extended region. 3C $138(=\mathrm{J} 0521+166)$ is associated with an $m_{v}=18.84$ quasar at redshift $z=0.759$ (Hewitt \& Burbridge 1989). Assuming $H_{0}=70, \Omega_{M}=0.3$ and $\Omega_{\Lambda}=0.7,1$ mas corresponds to a projected linear size of $7.4 \mathrm{pc}$. In this paper, we report on the results of a monitoring 
Table 1. VLA measurements of calibrators.

\begin{tabular}{llllr}
\hline \hline Date & Source & $S_{4.9} \mathrm{Jy}$ & $p$ mJy & $\chi$ \\
\hline 8 Aug. 1997 & 3C 84 & 24.3 & & \\
8 Aug. 1997 & B 0235+164 & 0.645 & 4.8 & 14 \\
8 Aug. 1997 & B 0016+731 & 0.790 & 13.7 & 63 \\
14 Sep. 1998 & BL Lac & 3.88 & 148.5 & -9 \\
14 Sep. 1998 & B 0235+164 & 2.956 & 14.0 & 2 \\
\hline
\end{tabular}

program probing the fine scale structure of the Faraday screen near the nucleus of this quasar.

\section{Observations and data reduction}

In order to monitor the Faraday screen in front of the inner jet in 3C 138, a series of observations was carried out using the VLBA plus a single VLA antenna. The epochs and frequencies observed are summarized in Table 2. The 1994 observations have been previously described in Cotton et al. (1997). Observations of 3C 138 were interspersed with calibrator observations: fringe finder (3C 84), instrumental polarization (3C 84, B 0016+731), polarization angle calibrator (B 0235+164, BL Lac, 3C 286, 4C 39.25). The 1997 and 1998 sessions were calibrated using short, nearly simultaneous measurements of the calibrators by the full VLA using $2 \times 50 \mathrm{MHz}$ bands centered at 4.8851 and $4.8351 \mathrm{GHz}$. These measurements used 3C 48 and 3C 138 as flux density and polarization calibrators using standard values and are described in Table 1. The first column in this table is the date of observation, the second is the source name, the third the total flux density at $4.9 \mathrm{GHz}$, the fourth, the linearly polarized intensity and the final column gives the derived integrated polarization angle of the source.

VLA calibration time was not available for sessions after 1998 and the standard VLA monitoring of flux densities and polarization angles was used. The VLA "standard monitoring" uses calibration done during normal operations, and observations of a given calibrator are sporadic and unpredictable. This means that the calibrator observations are generally further from the epoch of the observations than when the dedicated VLA calibration sessions are available and source variability degrades the accuracy of the calibration. The best external calibration was obtained for the 1998 observations and the polarization angle calibration at other epochs were referred to this epoch using the (assumed) constant polarization in the outer jet.

All processing was done in the NRAO AIPS package following standard procedures as outlined in Cotton (1993). Visibilities were calibrated by applying measured system temperatures and "phase cals". After calibrators were fringe fitted and self-calibrated, any amplitude corrections needed to correct to VLA integrated flux densities were applied. Instrumental polarization was determined in each $8 \mathrm{MHz}$ band separately as ellipticity and orientation from unpolarized calibrators. Observations of 3C 138 were fringe fitted, amplitude and polarization corrected and then phase self calibrated with all epochs starting from a common model and using a common procedure performed using the same software. Due to the wide
Table 2. Epoch and frequencies of the observations.

\begin{tabular}{lrr}
\hline \hline Date & Epoch & $\begin{array}{r}\text { Frequencies } \\
\mathrm{GHz}\end{array}$ \\
\hline 19 December 1994 & $\mathrm{A}$ & $4.7,5.0$ \\
6 August 1997 & $\mathrm{B}$ & $4.680,4.688,4.987,4.995$ \\
11 September 1998 & $\mathrm{C}$ & $4.680,4.688,4.987,4.995$ \\
6 August 2000 & $\mathrm{D}$ & $4.680,4.688,4.987,4.995$ \\
14 October 2002 & $\mathrm{E}$ & $4.680,4.688,4.987,4.995$ \\
\hline
\end{tabular}

Table 3. RMS off-source noise in images.

\begin{tabular}{lcccc}
\hline \hline & $4.7 \mathrm{GHz}$ & $4.7 \mathrm{GHz}$ & $5.0 \mathrm{GHz}$ & $5.0 \mathrm{GHz}$ \\
Epoch & $I$ & $Q, U$ & $I$ & $Q, U$ \\
& $\mathrm{mJy}$ & $\mathrm{mJy}$ & $\mathrm{mJy}$ & $\mathrm{mJy}$ \\
\hline $\mathrm{A}$ & 0.30 & 0.21 & 0.30 & 0.11 \\
$\mathrm{~B}$ & 0.21 & 0.10 & 0.20 & 0.10 \\
$\mathrm{C}$ & 0.24 & 0.15 & 0.21 & 0.14 \\
$\mathrm{D}$ & 0.29 & 0.11 & 0.26 & 0.11 \\
$\mathrm{E}$ & 0.22 & 0.10 & 0.21 & 0.10 \\
\hline
\end{tabular}

spanned bandwidth of the observations and the spectral index variations across the source, the data were self calibrated and imaged independently in the 4.7 and $5.0 \mathrm{GHz}$ bands. Data were only averaged to $2 \mathrm{MHz}$ prior to imaging to reduce the effects of chromatic aberration. The extended portion of the jet is very heavily resolved which complicates the deconvolution. In order to improve the deconvolution, a multi resolution CLEAN was used with three resolutions with linear sizes one, three and nine times the full resolution. This procedure made a very significant improvement in the quality of the extended portions of the images.

Final polarization angle corrections were derived from the highly polarized end of the jet in $3 \mathrm{C} 138$ by comparing the polarization images at each epoch with that derived from the reference epoch, September 1998. Prior to comparison, the images were aligned on component "A", the location of the nucleus (Cotton et al. 1997). The polarization angle corrections were determined by a polarized flux density weighted average of the difference of polarization angles between each epoch and the reference epoch. The scatter in the results give an RMS uncertainty of $0.5^{\circ}$ in the relative polarization calibration. This procedure should insure that any residual systematic rotation measure offset is common to all observations and the polarization angles should have relative alignment errors of $0.5^{\circ}$ or less between the images made at different epochs. The 4.7 and $5.0 \mathrm{GHz}$ corrections also agreed at the $0.5^{\circ}$ level so any residual systematic rotation measure should be less than $18 \mathrm{rad} \mathrm{m}^{-2}$.

\section{Results}

Data from each epoch were imaged in the same fashion using a common restoring beam $\left(2.5 \times 1.3\right.$ mas at $\left.\mathrm{PA}=25^{\circ}\right)$. In order to determine rotation measures, data around 4.7 and $5.0 \mathrm{GHz}$ were imaged separately. The off-source RMS noise values in the various images are given in Table 3; the first column in this table is epoch designation; following this are the RMS noise values for 


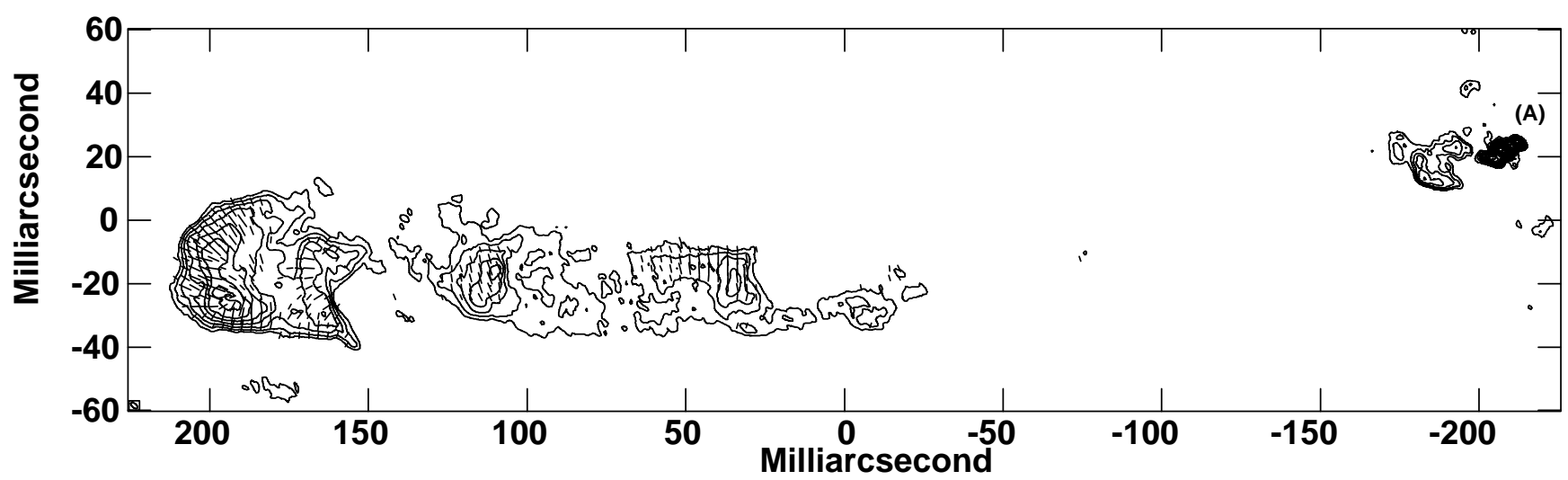

Fig. 1. Contour plot of the average image of 3C 138 with superposed polarization "E" vectors. Vector length is proportional to polarized intensity. The lowest contour is $0.5 \mathrm{mJy}$ and successive contours are factors of $\sqrt{2}$ larger. The core (A) is on the upper right. This image is rotated on the sky by $25^{\circ}$.

Table 4. Polarized components.

\begin{tabular}{|c|c|c|c|c|c|c|c|c|c|c|c|c|}
\hline \multirow{3}{*}{ Epoch } & $\mathrm{B}_{1}$ & $4.7 \mathrm{GHz}$ & & $\mathrm{B}_{1}$ & $5.0 \mathrm{GHz}$ & & $\mathrm{B}_{2}$ & $4.7 \mathrm{GHz}$ & & $\mathrm{B}_{2}$ & $5.0 \mathrm{GHz}$ & \\
\hline & $I$ & $P$ & $x$ & $I$ & $P$ & $x$ & $I$ & $P$ & $\chi$ & $I$ & $P$ & $x$ \\
\hline & $\mathrm{mJy}$ & mJy & $\circ$ & $\mathrm{mJy}$ & mJy & $\circ$ & $\mathrm{mJy}$ & mJy & $\circ$ & $\mathrm{mJy}$ & mJy & $\circ$ \\
\hline A & 134 & 5.0 & $-37(1.2)$ & 134 & 4.7 & 11(1.2) & 8 & 0.4 & $-45(15)$. & 8 & 0.8 & $-39(7.2)$ \\
\hline B & 143 & 3.0 & $-8(1.1)$ & 116 & 2.8 & $35(1.1)$ & 18 & 0.3 & $-70(11)$. & 13 & 0.5 & $-38(5.5)$ \\
\hline $\mathrm{C}$ & 133 & 3.5 & $-18(1.3)$ & 125 & 4.0 & $27(1.1)$ & 15 & 0.8 & $-32(5.6)$ & 15 & 1.2 & $-20(3.4)$ \\
\hline D & 98 & 2.4 & $-4(1.4)$ & 73 & 2.2 & $42(1.4)$ & 20 & 1.2 & $-52(2.7)$ & 22 & 1.2 & $-16(2.6)$ \\
\hline $\mathrm{E}$ & 84 & 1.9 & $-37(1.5)$ & 80 & 2.1 & $10(1.4)$ & 24 & 1.5 & $-56(2.0)$ & 21 & 1.7 & $-25(1.7)$ \\
\hline
\end{tabular}

the $I$ and $Q, U$ images at 4.7 and $5.0 \mathrm{GHz}$. The average of the images of the 1997-2002 data is shown in Fig. 1 with overlaid polarization vectors. The core is to the upper right of the figure. The resulting images of the region of the inner jet are shown in Fig. 2. The polarized emission in the 4.7 and $5.0 \mathrm{GHz}$ images is shown in Fig. 3. This figure shows the emergence of a second polarized component in the September 1998 data. The component designations used in the following are shown in Figs. 2 and 3 . The total intensity, linearly polarized intensities and polarization angles for the two polarized components in the inner jet at 4.7 and $5.0 \mathrm{GHz}$ are shown in Table 4. Estimated errors are shown in tables in parentheses following the value.

The Faraday rotation of the emission from the two polarized components was estimated from the Stokes' $Q$ and $U$ images derived from the 4.7 and $5.0 \mathrm{GHz}$ data. This two frequency measurement of the variation of polarization angle with frequency cannot demonstrate the expected $\lambda^{2}$ behavior of Faraday rotation so it must be assumed that Faraday rotation is the cause of any difference in the polarization angle. The resulting inferred rotation measures are given in the observer's frame in Table 5 and in Fig. 4. The first column in Table 5 is the epoch designation for this session, the second column is the Faraday rotation derived for Component $\mathrm{B}_{1}$ (see Fig. 3), the third column is the fractional linear polarization of Component $\mathrm{B}_{1}$, the fourth column is the Faraday rotation derived for Component $\mathrm{B}_{2}$ and the final column the fractional linear polarization of Component $\mathrm{B}_{2}$. The possibility of multiple turns of polarization angle was ruled out by a comparison of the polarization angles of the closely spaced bands near 4.7 and $5.0 \mathrm{GHz}$.

If all variations in polarization angle can be assumed to be due to variations in rotation measure, then a more sensitive measure of the variations in time of the Faraday rotation can be made. These estimates of the change in rotation measure relative to the August 2000 epoch are shown for the 4.7 and $5.0 \mathrm{GHz}$ data in Table 6 as well as Fig. 4. The first column in Table 6 is the epoch designation, followed by the differential Faraday rotations of Component $\mathrm{B}_{1}$ estimated at 4.7 and $5.0 \mathrm{GHz}$; the final columns are the differential Faraday rotations of Component $\mathrm{B}_{2}$ estimated at 4.7 and 5.0 GHz. The good correlation of the relative rotation measure determined by this method and from the difference in the polarization angles for the earlier epochs suggest that most of the variations are due to variable Faraday rotation rather than intrinsic variations in the emission.

The final two epochs showed increasing resolution of the polarized emission, which is probably the principle cause of the disagreement between the differential and total rotation measures. Even though the same restoring beam was used for all images, the intrinsically lower resolution of the $4.7 \mathrm{GHz}$ data will cause a greater blending of different regions of emission which will degrade the reliability of the Faraday rotation derived from comparing images at the two frequencies. Since the differential Faraday rotation is determined from differences in the polarization angle measured at the same frequency (and 

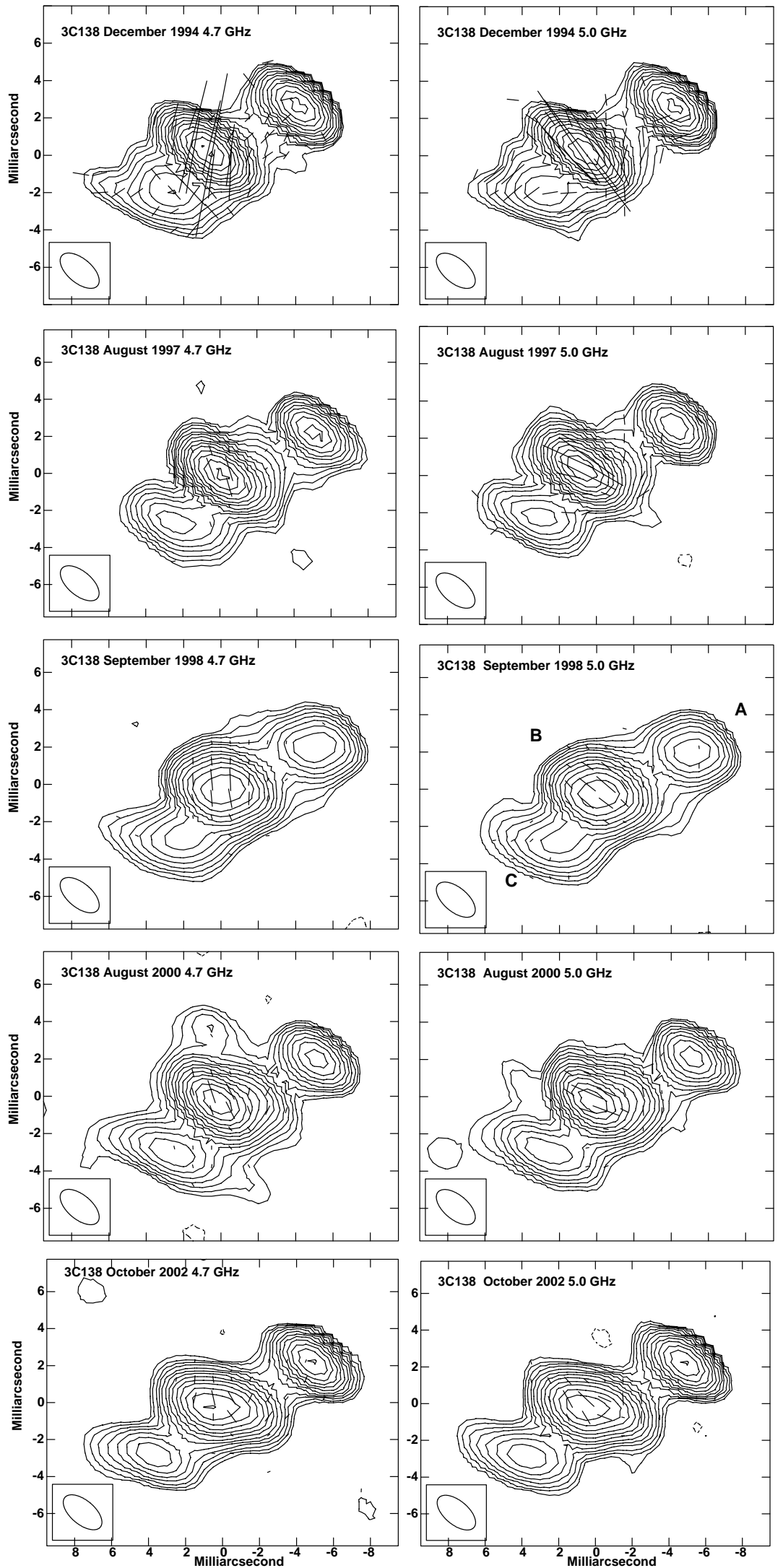

Fig. 2. Total intensity contours (powers of $\sqrt{2}$ times $1.2 \mathrm{mJy} /$ beam) of the core and inner jet of $3 \mathrm{C} 138$ with vectors proportional to polarized intensity and with the orientation of the $\boldsymbol{E}$-vector. Images on the left are at $4.7 \mathrm{GHz}$ and on the right at $5.0 \mathrm{GHz}$. Component designations are shown in the September $19985.0 \mathrm{GHz}$ panel. Images are rotated on the sky by $25^{\circ}$ to align them with the large scale image in Fig. 1. 

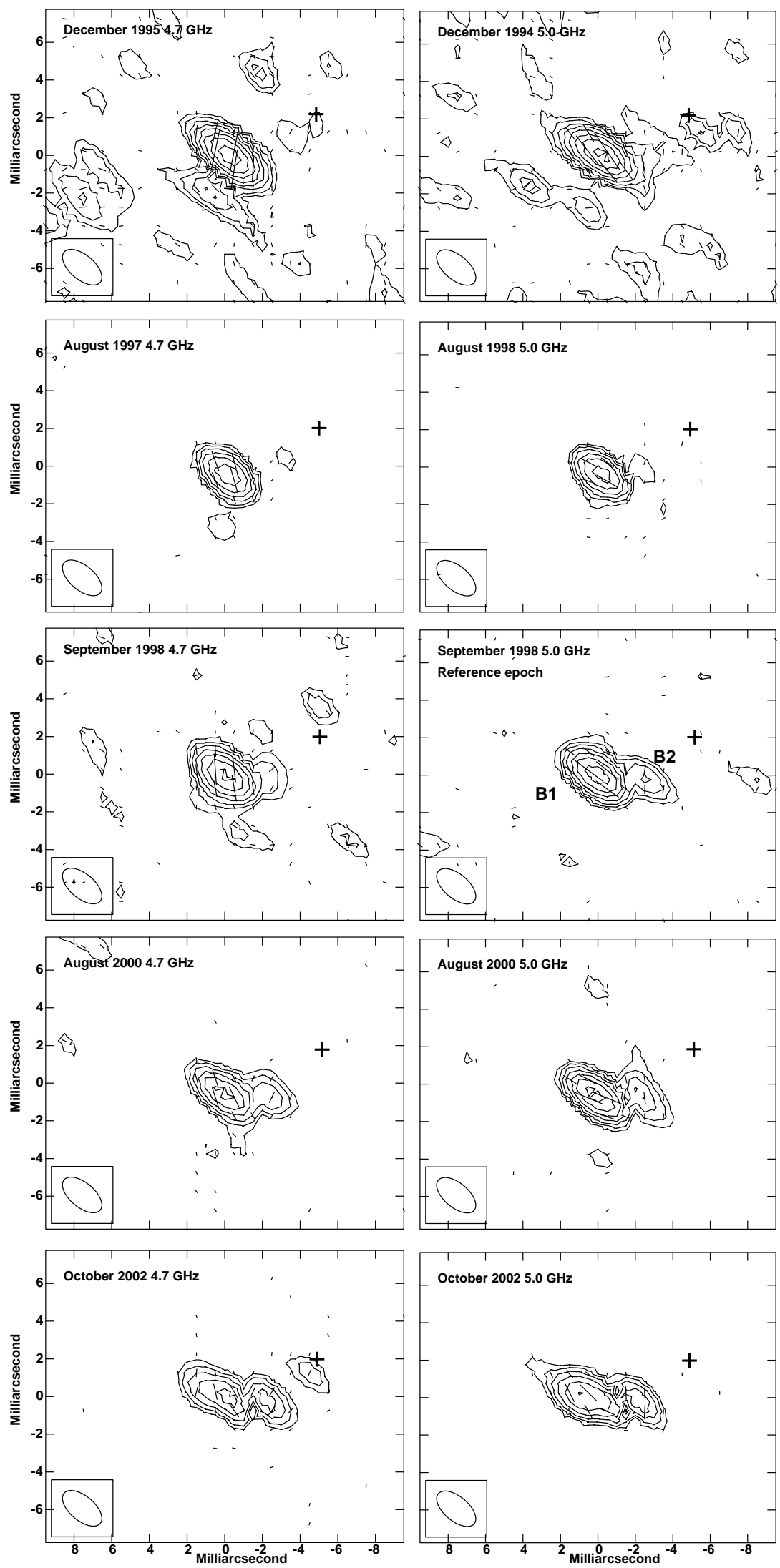

Fig. 3. Polarized intensity contours (powers of $\sqrt{2}$ times $0.3 \mathrm{mJy} / \mathrm{beam}$ ) of the core and inner jet of $3 \mathrm{C} 138$. Images on the left are at $4.7 \mathrm{GHz}$ and on the right at $5.0 \mathrm{GHz}$. The two polarized component designations are shown in the September $19985.0 \mathrm{GHz}$ panel. The location of the core is marked with a cross. Images are rotated on the sky by $25^{\circ}$ to align them with the large scale image in Fig. 1. 

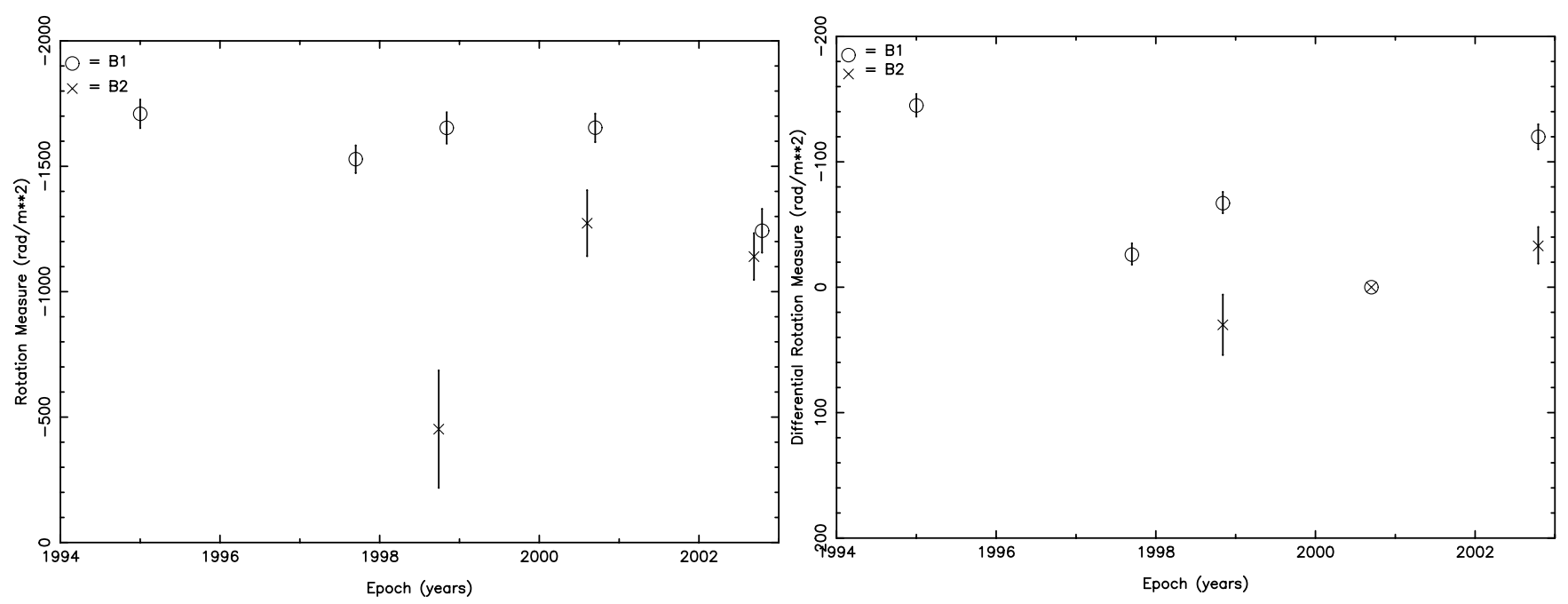

Fig. 4. Left: rotation measures of the two polarized features in the the inner jet determined from the 4.7 and $5.0 \mathrm{GHz}$ polarized images. Right: average differential rotation measures of the two polarized features determined from the difference of the polarization angle from that of the August 2000 epoch. All values displayed are in the observer's frame, to convert to the rest frame of the source, multiply by $(1+z)^{2}=3.09$.

Table 5. Faraday rotation measure and fractional polarization.

\begin{tabular}{cccrc}
\hline \hline Epoch & $\begin{array}{c}\mathrm{B}_{1} \\
\mathrm{rad} / \mathrm{m}^{2}\end{array}$ & $\%$ pol & $\begin{array}{r}\mathrm{B}_{2} \\
\mathrm{rad} / \mathrm{m}^{2}\end{array}$ & $\%$ pol \\
\hline $\mathrm{A}$ & $-1709(57)$ & $3.6(0.2)$ & $-220(620)$ & $6.8(2.5)$ \\
$\mathrm{B}$ & $-1528(55)$ & $1.9(0.1)$ & $-1140(420)$ & $2.8(0.7)$ \\
$\mathrm{C}$ & $-1652(62)$ & $2.9(0.1)$ & $-450(230)$ & $6.6(1.0)$ \\
$\mathrm{D}$ & $-1654(57)$ & $2.8(0.1)$ & $-1270(130)$ & $5.7(0.5)$ \\
$\mathrm{E}$ & $-1243(87)$ & $2.5(0.1)$ & $-1140(90)$ & $7.0(0.4)$ \\
\hline
\end{tabular}

Table 6. Differential Faraday rotation.

\begin{tabular}{lcccc}
\hline \hline Epoch & $\begin{array}{c}\mathrm{B}_{1} 4.7 \mathrm{GHz} \\
\mathrm{rad} / \mathrm{m}^{2}\end{array}$ & $\begin{array}{c}5.0 \mathrm{GHz} \\
\mathrm{rad} / \mathrm{m}^{2}\end{array}$ & $\begin{array}{c}\mathrm{B}_{2} 4.7 \mathrm{GHz} \\
\mathrm{rad} / \mathrm{m}^{2}\end{array}$ & $\begin{array}{c}5.0 \mathrm{GHz} \\
\mathrm{rad} / \mathrm{m}^{2}\end{array}$ \\
\hline $\mathrm{A}$ & $-140(8)$ & $-150(9)$ & & \\
$\mathrm{B}$ & $-17(8)$ & $-36(9)$ & & \\
$\mathrm{C}$ & $-63(8)$ & $-71(9)$ & $80(24)$ & $-20(16)$ \\
$\mathrm{D}$ & 0 & 0 & 0 & 0 \\
$\mathrm{E}$ & $-87(9)$ & $-154(10)$ & $-22(9)$ & $-44(8)$ \\
\hline
\end{tabular}

resolution), it is less susceptible to this effect, but is sensitive to changes in the intrinsic polarization angle.

The positions, sizes, and flux densities of the components in the inner jet were determined by a least squares procedure (AIPS program JMFIT). Component A is shown in Cotton et al. (1997) to have a flat spectrum and is assumed to indicate the nucleus. The separations of the components from Component A are given in Table 7 and shown in Fig. 5. The first column in Table 7 is the epoch designation followed by the offset of the Gaussian components fitted to components B and C from the position of Component A. Derived integrated flux densities are given in Table 8, and component upper limits to component sizes are shown in Table 9.
Table 7. Component offsets from the core (Comp. A).

\begin{tabular}{lcc}
\hline \hline Epoch & $\mathrm{B}$ & $\mathrm{C}$ \\
& mas & mas \\
\hline $\mathrm{A}$ & $5.365(.007)$ & $8.158(.037)$ \\
$\mathrm{B}$ & $5.430(.010)$ & $8.539(.027)$ \\
$\mathrm{C}$ & $5.478(.018)$ & $8.650(.027)$ \\
$\mathrm{D}$ & $5.704(.082)$ & $9.084(.055)$ \\
$\mathrm{E}$ & $5.670(.006)$ & $9.892(.022)$ \\
\hline
\end{tabular}

Table 8. Component integrated flux densities.

\begin{tabular}{lccc}
\hline \hline Epoch & $\begin{array}{c}\mathrm{A} \\
\mathrm{mJy}\end{array}$ & $\begin{array}{c}\mathrm{B} \\
\mathrm{mJy}\end{array}$ & $\begin{array}{c}\mathrm{C} \\
\mathrm{mJy}\end{array}$ \\
\hline $\mathrm{A}$ & $103.3(0.6)$ & $195.8(0.7)$ & $18.1(1.0)$ \\
$\mathrm{B}$ & $51.7(0.5)$ & $212.2(0.5)$ & $41.6(0.8)$ \\
$\mathrm{C}$ & $52.0(0.5)$ & $212.7(0.5)$ & $46.5(0.8)$ \\
$\mathrm{D}$ & $44.9(0.7)$ & $215.4(0.7)$ & $46.6(1.0)$ \\
E & $90.0(0.5)$ & $221.9(0.7)$ & $38.3(0.7)$ \\
\hline
\end{tabular}

Table 9. Component size upper limits.

\begin{tabular}{lccc}
\hline \hline Epoch & $\mathrm{A}$ & $\mathrm{B}$ & $\mathrm{C}$ \\
& mas & mas & mas \\
\hline A & $<2.0$ & $<2.2$ & $<4.0$ \\
B & $<2.2$ & $<2.3$ & $<3.9$ \\
C & $<2.0$ & $<2.3$ & $<3.4$ \\
D & $<2.1$ & $<2.4$ & $<4.0$ \\
E & $<2.0$ & $<3.2$ & $<3.6$ \\
\hline
\end{tabular}

The Gaussian fits to the total intensity images were unable to determine the extent of Component B; however, at the September 1998 and later epoch this component was well resolved in linear polarization into Components $\mathrm{B}_{1}$ and $\mathrm{B}_{2}$ (see Fig. 3). Visual inspection of Fig. 2 also suggests an extension 


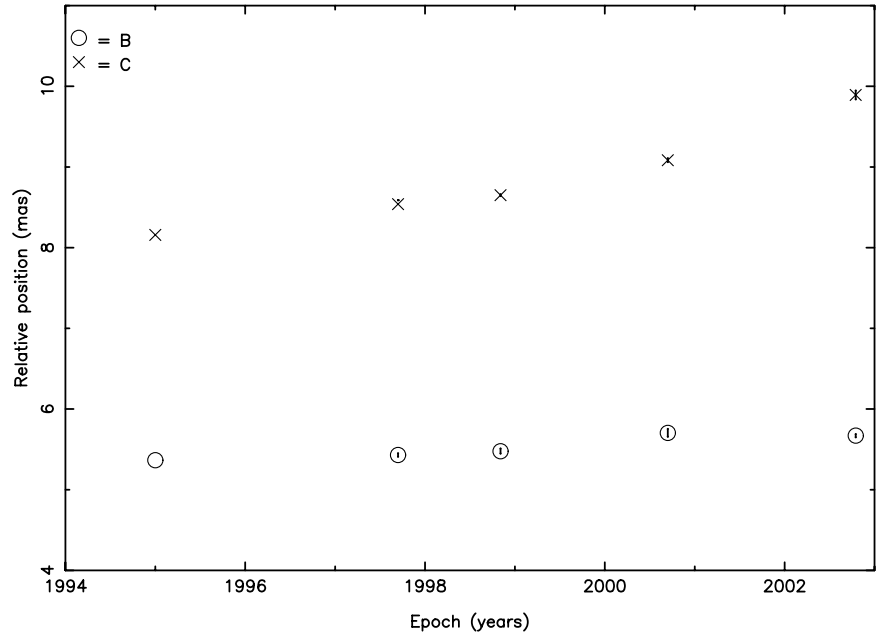

Fig. 5. Average offsets of the centers of the Gaussians fitted to Components B and C from Component A. Formal errors are smaller than the symbols plotted.

of Component $\mathrm{B}$ in the region of polarized Component $\mathrm{B}_{2}$. A combination of the slow total motion of Component $\mathrm{B}$ and the weak and resolved nature of the polarized component prevents the direct detection of motion in the polarized components. However, the peak in the linearly polarized emission of Component $\mathrm{B}_{1}$ is systematically offset from the peak of the total intensity Component B. In the coordinates displayed in Fig. 3, the offset in the " $X$ " direction was $1 \pm 80 \mu$ as and $180 \pm$ $30 \mu$ as in the " $Y$ " direction; the uncertainties being determined from the scatter in the values determined from different epochs and in the independent 4.7 and $5.0 \mathrm{GHz}$ measurements. Thus, the emission from Component $\mathrm{B}_{1}$ appears to be from the side of Component B closest to the core.

\section{Discussion}

The extended jet region in 3C 138 emits most of the observed flux density and this emission is strongly polarized. Thus, the changes measured in the weakly polarized core are a very small fraction of the total polarized emission. These small changes in the polarized emission are consistent with the observed relatively constant integrated polarized emission and the widespread use of 3C 138 as a polarization calibrator.

During the period of the observations reported here, the motion of Component B has slowed to an undetectable level between the last two epochs whereas the motion of Component $C$ has increased (see Fig. 5). As the motion of Component B slows, a second polarized component becomes more prominent. The presence of substructure in this component complicates the interpretation of motion. There is no direct evidence for relative motion of Components $\mathrm{B}_{1}$ and $\mathrm{B}_{2}$. The October 2002 observations (Fig. 3) suggest the emergence of a third component on the opposite side of Component $\mathrm{B}_{1}$ from $\mathrm{B}_{2}$.

The presence of two (or more), closely spaced polarized components seen in Fig. 3 complicates the measurement of Faraday rotation. The same restoring beam was used for all images but the $4.7 \mathrm{GHz}$ data has intrinsically lower resolution than the $5.0 \mathrm{GHz}$ data. Since linear polarization is a vector quantity and the polarization angles of the two components are quite different, the emission does not simply add as does total intensity. The mutual influence of the two components will differ at the two frequencies, distorting the derived value of the Faraday rotation. If the background illuminating source has constant polarization angle then variations in the observed polarization angle are a more sensitive indicator of Faraday rotation than the differences in polarization angles with frequency and are somewhat less sensitive to the effects of multiple components. The good correlation of the total and differential Faraday rotation measures in the period before Component $\mathrm{B}_{2}$ became prominent supports the use of the differential Faraday rotation.

In spite of the slow motion of Component B, the Faraday rotation monitoring results shown in Fig. 4 indicate low level variations in rotation measure. This is seen more clearly in the case of the differential Faraday rotation which is more precise but assumes that the intrinsic polarization angle is constant. The short time span over which the rotation measure of Component $\mathrm{B}_{2}$ and the weakness of this component prevent the detection of time variations in this components but it clearly differs from $B_{1}$. The fluctuations in rotation measure appear to be stochastic rather than systematic. The values given in Fig. 4 and Tables 5 and 6 are calculated in our frame, in the rest frame of the source they must be corrected by $(1+z)^{2}$ or by a factor of 3.09 , giving a typical value for Component $\mathrm{B}_{1}$ of $\approx-5000 \mathrm{rad} / \mathrm{m}^{2}$

Assuming Component $\mathrm{B}_{1}$ is fixed relative to Component $\mathrm{B}$, it has moved $\approx 330 \mu$ as $(\approx 2.4$ pc projected) during these observations. Component $\mathrm{B}_{1}$ showed $4 \%$ RMS fluctuations over this period. Component $\mathrm{B}_{2}$ is $2000 \mu \mathrm{as}(14.4 \mathrm{pc})$ away but closer to the nucleus yet has a $25 \%$ lower rotation measure and much higher fractional polarization. These suggest that the Faraday screen has structure on the pc and possibly the sub-pc scale.

The systematic offsets of the peaks in linearly polarized emission from that of total intensity suggest a "holes in a screen" scenario. In this scenario, the "holes" would still have a substantial Faraday rotation but would be surrounded by a medium of very large Faraday depth. The fluctuations in the polarized intensities in Components $\mathrm{B}_{1}$ and $\mathrm{B}_{2}$ are strongly correlated with the variations in total intensity at the same location (Table 4) resulting in approximately constant fractional polarizations (Table 5) for both components; this is also consistent with holes in a screen illuminated by a background source. The lack of detectable polarized emission in Component $\mathrm{C}$ are also consistent with a patchy but dense Faraday screen; fractional polarization as large as seen in Component $\mathrm{B}_{2}$ would be easily detectable in Component $\mathrm{C}$ which is much further from the nucleus.

In a simple "holes in a screen" model, the holes through which the emission emerges would be stationary and therefore the emission emerging from these holes would have a constant Faraday rotation. However, Fig. 4 shows significant variation in at least differential Faraday rotation; this is equivalent to the variations in the polarization angle seen in Table 4 and Figs. 2 and 3. The timescale of these fluctuations is too short to be due to gas motions in the ISM and are more likely associated with the motion of the illuminating components. This suggests 
the possibility that these Faraday rotation variations are due to the interaction of the jet and the ISM of the host galaxy; for example, variable shock ionization of neutral gas impacted by the jet.

\section{Conclusions}

During the period of the observations reported here, the motion of the polarized component in the inner jet of $3 \mathrm{C} 138$ has slowed to an undetectable level. This component has a large Faraday rotation and in spite of its slow movement, variations of Faraday rotation are detected. In September 1998 and later observations a second polarized sub-component is clearly seen. Faraday rotation variations in time and space are inconsistent with a simple, monotonic decrease in Faraday depth with increasing distance from the nucleus but require a more irregular medium.

The projected distance of the polarized component from the core is $40 \mathrm{pc}$; the centroid of this component moved by $2.5 \mathrm{pc}$ during the 7.8 years covered by these observations although the motion slowed dramatically. There is another, weaker component at a projected distance of $65 \mathrm{pc}$ from the core from which polarized emission is not detected. This outer component appeared to accelerate during the observations and moved by $12.8 \mathrm{pc}$.

The offset of the peak of polarized emission from the total intensity peak, as well as the correlation of polarized with total intensity, strongly suggest that the polarized emission is from relatively small holes in an otherwise dense Faraday screen. If this be the case, the variation in Faraday rotation are not the result of a background source moving behind a variable
Faraday screen but is more likely the result of jet-ISM interactions. Variable shock ionization of the ISM by the jet is a possibility.

Acknowledgements. The authors would like to thank the referee, A. Tzioumis, for comments leading to the improvement of the presentation of the paper.

\section{References}

Axon, D. J., Capetti, A., Fanti, R., et al. 2000, AJ, 120, 2284

Cotton, W. D. 1993, AJ, 106, 1241

Cotton, W. D., Dallacasa, D., Fanti, C., et al. 1997, A\&A, 325, 493

Flatters, C. 1998, in IAU Colloq. 164: Radio Emission from Galactic and Extragalactic Compact Sources, ed. J. A. Zensus, G. B. Taylor, \& J. M. Wrobel (Astronomical Society of the Pacific), ASP Conf. Ser., 144, 109

Gelderman, R., \& Whittle, M. 1994, ApJS, 91, 491

Hewitt, A., \& Burbridge, G. 1989, ApJS, 69, 1

Mantovani, F., Junor, W., Ricci, R., et al. 2002, A\&A, 389, 58

Nan, R., Gabuzda, D. C., Kameno, S., Schilizzi, R. T., \& Inoue, M. 1999, A\&A, 344, 402

O’Dea, C. P. 1998, PASP, 110, 493

Peacock, J. A., \& Wall, J. V. 1982, MNRAS, 198, 843

Shen, Z. Q., Jiang, D. R., Kameno, S., \& Chen, Y. J. 2001, A\&A, 370, 65

Taylor, G. B., Venturi, T., \& Udomprasert, P. S. 1998, in IAU Colloq. 164: Radio Emission from Galactic and Extragalactic Compact Sources, ed. J. A. Zensus, G. B. Taylor, \& J. M. Wrobel (Astronomical Society of the Pacific), ASP Conf. Ser., 144, 1113

Zavala, R. T., \& Taylor, G. B. 2001, ApJ, 550, L147

Zavala, R. T., \& Taylor, G. B. 2002, ApJ, 566, L9 